\title{
Comparison between adaptive and adjustment procedures for binaural loudness balancing
}

\author{
Maaike Van Eeckhoutte, Dimitar Spirrov, and Tom Francart ${ }^{\text {a) }}$ \\ Department of Neurosciences, ExpORL, KU Leuven, Leuven, Belgium
}

(Received 7 December 2017; revised 30 May 2018; accepted 1 June 2018; published online 29 June 2018)

\begin{abstract}
Binaural loudness balancing is performed in research and clinical practice when fitting bilateral hearing devices, and is particularly important for bimodal listeners, who have a bilateral combination of a hearing aid and a cochlear implant. In this study, two psychophysical binaural loudness balancing procedures were compared. Two experiments were carried out. In the first experiment, the effect of procedure (adaptive or adjustment) on the balanced loudness levels was investigated using noise band stimuli, of which some had a frequency shift to simulate bimodal hearing. In the second experiment, the adjustment procedure was extended. The effect of the starting level of the adjustment procedure was investigated and the two procedures were again compared for different reference levels and carrier frequencies. Fourteen normal hearing volunteers participated in the first experiment, and 38 in the second experiment. Although the final averaged loudness balanced levels of both procedures were similar, the adjustment procedure yielded smaller standard deviations across four test sessions. The results of experiment 2 demonstrated that in order to avoid bias, the adjustment procedure should be conducted twice, once starting from below and once from above the expected balanced loudness level. (C) 2018 Acoustical Society of America.
\end{abstract}

https://doi.org/10.1121/1.5042522

$[\mathrm{VB}]$

Pages: $3720-3729$

\section{INTRODUCTION}

Binaural loudness balancing is important for the fitting of bilateral hearing aids, bilateral cochlear implants, and in particular for bimodal listeners. This steeply growing population has a cochlear implant in one ear and a hearing aid in the other, contralateral ear. Psychophysical procedures to find the balanced loudness across the two ears are also commonly used in research. Here, we define binaural loudness balance as follows: in those cases where the binaural stimulus results in a single fused auditory image, we define it as binaurally balanced if the single image is perceived (lateralized) in the center of the head. In those cases where the binaural stimulus does not result in a single fused image, but in two separate images, we define it as binaurally balanced if the two separate sound images are perceived as equally loud. We expect that normal hearing listeners perceive a single fused sound image for correlated sounds, and that binaural balance is achieved for an interaural level difference (ILD) of $0 \mathrm{~dB}$ (Akeroyd, 2006), but for hearing impaired listeners this may not be the case.

Benefits associated with a fitting that results in a balanced percept for stimuli originating from the front have been shown in many studies. For example, bilateral cochlear implant users mainly rely on ILDs for sound source localization (Salloum et al., 2010). Therefore, a perceived balanced loudness between both implant sides should improve the localization skills of these users. For bimodal listeners, advantages for speech understanding and comfort have been

\footnotetext{
${ }^{\text {a)} E l e c t r o n i c ~ m a i l: ~ t o m . f r a n c a r t @ m e d . k u l e u v e n . b e ~}$
}

described when both devices are loudness balanced (Ching et al., 2007; Dorman et al., 2014; Tyler et al., 2002).

Due to the differences between the cochlear implant and the hearing aid side, bimodal listeners often experience a tonotopical or frequency mismatch (Francart and McDermott, 2013). The frequency mismatch results in decreased fusion from the two devices (Guérit et al., 2014; Reiss et al., 2016; Tan et al., 2017). Most probably, some bimodal listeners can adapt to frequency mismatches, while others cannot (Francart and McDermott, 2013; Tan et al., 2017). However, with increasing frequency mismatch, just noticeable differences (JNDs) in ILD become larger (Francart and Wouters, 2007). Since bimodal listeners have larger JNDs in ILD than normal hearing listeners (Francart et al., 2008a), we can assume that the range of levels at which bimodal listeners experience equal loudness might be larger or at least more variable compared to normal hearing listeners. Therefore, the result of the loudness balancing procedure will also be more variable.

Several psychophysical procedures have been described for binaural loudness balancing. Using the method of constants, a wide range of levels is chosen in advance by the experimenter and is presented to the comparison ear in random order, while the level of the reference ear is fixed. Consequently, the complete psychometric function is measured, which is time consuming while only the $50 \%$ point of the curve is needed for loudness balancing (Fletcher and Munson, 1933; Jesteadt, 1980; Levitt, 1971).

To improve efficiency, in a simple adjustment procedure, also known as the method of limits, a faster estimate of the $50 \%$ point is found by manually increasing or decreasing the stimulus level until the participant's responses demonstrate a reversal. For example, to estimate the hearing 
threshold, the stimulus intensity is decreased until the participant cannot hear the stimulus, or to estimate the balanced loudness, the stimulus intensity of the comparison ear is increased until the participant reports equal loudness across the two ears, while the intensity of the reference ear remains the same. A disadvantage of this procedure is that it can be substantially biased due to sequential effects, given that the stimuli are presented in an ordered series (Jesteadt, 1980; Levitt, 1971). Furthermore, the procedure strongly depends on the subject's response biases. They might be too quickly satisfied with the final outcome or may not be aware that they can perceive even more subtle differences (van Wieringen and Wouters, 2001).

Furthermore, the subjects expect that each category will be presented almost equally often (Stevens and Galanter, 1957). Therefore, they might attempt to use each category equally often. To avoid response biases due to sequential effects and the tendency to use each response category equally often, adaptive methods have been described in which the next stimulus is determined by the response on a previous trial. The participant has a forced choice between some alternative subjective response categories, e.g., the participant has to indicate whether the sound is "louder" or "softer" than the reference stimulus. The difficulty is often increased when the test proceeds. Instead of terminating after the first reversal, the adaptive procedure continues until several reversals are obtained.

To prevent the participant from noticing a pattern in the procedure, two interleaved independent sequences with different symmetric decision rules can be implemented, such that, e.g., one sequence starts at a point well above the balanced loudness percept and one sequence starts at a point well below this percept. Since the balanced loudness percept is usually not known, as for listeners with an asymmetric hearing loss, the run often starts far away from the balanced loudness. The start point of the sequence has been shown to have no effect on the final outcome (Jesteadt, 1980; Levitt, 1971). Large step sizes are used to quickly approximate the balanced loudness in the beginning, which are gradually reduced as the test continues.

In general, the adjustment and adaptive procedure are the most commonly used procedures for binaural loudness balancing. These procedures have also been used to measure the detection and discrimination thresholds of hearing and vision. van Wieringen and Wouters (2001) reported that both procedures were precise and feasible for hearing threshold determination in cochlear implant users and could be measured within a reasonable time span. However, the adaptive procedure took significantly more time than the adjustment procedure, while the latter showed more variability with inexperienced participants, as reflected by a larger testretest variation. The duration of the adaptive procedure can be reduced by using a simple one-up, one-down decision rule (converging to $50 \%$ on the psychometric curve) instead of other decision rules (e.g., one-up, two-down, converging to $71 \%$, which is suitable for threshold determination but not for loudness balancing). For threshold determination, no statistically significant differences were found between the outcome results for different percent correct points on the psychometric curve (e.g., 50\%, 70\%, and 79\%) (Lecluyse and Meddis, 2009; van Wieringen and Wouters, 2001). Under some conditions, the adaptive and adjustment procedures can produce different results. For example, in Wier et al. (1976), the adjustment procedure yielded significantly better frequency discrimination performance than the adaptive procedure. Although the adjustment and adaptive procedures have been described in the past for detection and discrimination thresholds, to our knowledge, the reliability of both procedures for loudness balancing has never been investigated.

The aim of this study was to (1) compare the adaptive with the adjustment procedure to measure binaural loudness balance with regard to repeatability and test duration, and (2) to investigate the influence of a frequency mismatch and thus of a non-fused sound image on the results.

Two experiments were carried out to answer these questions. In the first experiment, stimuli with and without interaural frequency shifts were loudness balanced using the adaptive and adjustment procedure in normal hearing participants. In a second experiment, we further explored the effect of procedure, start level, carrier frequency, and reference level using a condition without interaural frequency shifts.

\section{EXPERIMENT 1}

\section{A. Methods}

\section{Apparatus}

The experiment was part of a project approved by the medical ethical committee of the University Hospital Leuven. The experiment was performed in a quiet room, and the software platform APEX 3 (Francart et al., 2008b) was used. All stimuli were created in MATLAB R2013a (The MathWorks, Inc., Natick, MA) and were presented binaurally to the participants through Sennheiser HDA 200 headphones, which were connected to an RME Multiface II sound card (Audio AG, Haimhausen, Germany). The stimuli were calibrated in $\mathrm{dB}$ A using a Brüel \& Kjær coupler type 4153, and were band passed filtered white noise. All stimuli had a duration of $1 \mathrm{~s}$, with a rise and fall time of $200 \mathrm{~ms}$. The stimuli were stored as 16 bits wav-files with a sampling frequency of $44100 \mathrm{~Hz}$.

\section{Participants}

The participants were 14 healthy volunteers (eight female, six male), with an average age of $21 \pm 2$ years. All participants had normal hearing in both ears, defined as detection thresholds of $25 \mathrm{~dB}$ hearing level (HL) or better for all octave frequencies between 250 and $8000 \mathrm{~Hz}$, as assessed by pure tone audiometry, except for one participant, who had a threshold of $30 \mathrm{~dB} \mathrm{HL}$ at $8000 \mathrm{~Hz}$ in the left ear. The normal hearing was further confirmed by a digit triplet speech in noise test. It showed speech reception thresholds of $-8 \mathrm{~dB}$ signal-to-noise (SNR) or better, which is within the normal range (Jansen et al., 2013). 


\section{Stimuli}

A noise band filtered between 2 and $4 \mathrm{kHz}$ was always presented to the left, designated as the reference ear at a fixed intensity of $60 \mathrm{~dB}$ A. This represents a probable fitting situation where the volume of one device is fixed and the volume of the contralateral device is varied. To the right, designated as the comparison ear, one out of four different noise stimuli were presented, simultaneously:

- "Correlated noise": exactly the same noiseband as the one presented to the reference ear, filtered between 2 and $4 \mathrm{kHz}$.

- "Uncorrelated noise": another noiseband filtered between 2 and $4 \mathrm{kHz}$; this was done by regenerating the stimulus from a new sample of white noise.

- "Small frequency shift": a noiseband filtered between 1 and $2 \mathrm{kHz}$.

- "Big frequency shift": a noiseband filtered between 0.5 and $1 \mathrm{kHz}$.

The frequency mismatches simulated possible tonotopic frequency mismatches in the bimodal stimulation. All stimuli were created with a custom MATLAB script that used finite impulse response band pass filters with 1024 taps. The slopes were always equal to or bigger than $132 \mathrm{~dB} /$ octave.

\section{Procedures}

First, an adjustment procedure was conducted. The starting level of the stimulus in the comparison ear was $60 \mathrm{~dB} A$, which was the same as the reference level. This level was chosen because it results in comfortable loudness. Depending on the fitting and some processing options, hearing aid and cochlear implant listeners estimate levels from approximately 60 to $65 \mathrm{~dB}$ sound pressure level (SPL) as comfortably loud (Hoth, 2007; Scollie et al., 2010). This is also the case for normal hearing listeners (Oetting et al., 2014). The influence of the starting level of the adjustment procedure was further investigated in experiment 2 . The participants could click on the buttons "«," "«," "<," ">," "》," and "》" to change the intensity in the comparison ear with steps of $-6,-3,-2,+2,+3$, and $+6 \mathrm{~dB}$, respectively. They were instructed to start with the larger step sizes and to continue with the smaller ones to find balanced loudness between the ears. The participants were instructed that if the sound percept was fused, they had to center the sound image in the middle of the head. Otherwise, they had to make the sounds in the left and the right ear equally loud. After reaching this balanced loudness percept, the participants were encouraged to further explore the balanced percept by pressing the arrows associated with the smaller step sizes, and only to stop when they were sure that the sound was balanced.

Second, a two-alternative forced-choice (2AFC) adaptive procedure without feedback was conducted (Jesteadt, 1980). To avoid response bias, two interleaved sequences using 2-down 1-up and 1-down 2-up decision rules were used instead of a simpler 1-down 1-up procedure where stimulus level is symmetrical around the estimation level (Levitt, 1971). Also, this procedure can be used for JND estimation (see below). To make sure the starting value was well above the balanced loudness, the result of the adjustment procedure $\pm 9 \mathrm{~dB}$ was used as the comparison ear intensity starting value for the sequences with a 2-down 1up, and a 1-down 2-up decision rule, converging to $71 \%$ and $29 \%$ correct. The initial step size was $6 \mathrm{~dB}$. After two and four reversals it was changed to 3 and $2 \mathrm{~dB}$, respectively. The final step size was chosen to be approximately two times larger than the JND in ILD of normal hearing subjects (Mills, 1960; Yost and Dye, 1988). Since each sequence had 25 trials, the total procedure consisted of 50 trials. For each sequence, the average intensity in $\mathrm{dB}$ of the last 12 trials was calculated, and the final result of the adaptive procedure was calculated as the average of these values of both sequences. For termination of the procedure and calculation of the result, we used trials instead of reversals because Monte Carlo simulations showed that independently of the slope of the psychometric function, using the last 12 trials results in smaller standard deviation across all simulations than using the last four reversals. More information is provided in the Appendix.

Before the start of the test phase, both adjustment and adaptive procedures were first included in a training phase with correlated noise to make the participants familiar with the task. As in the test phase, the adjustment procedure was always first because we needed its result for the start levels of the adaptive procedure. The results of the training phase were not used for the analysis. To investigate test-retest repeatability, four loudness balancing tests were administered. Test sessions A1 and A2 were administered on the same day, with a $10 \mathrm{~min}$ break between both tests. Similarly, sessions B1 and B2 were administered one week after sessions $\mathrm{A} 1$ and $\mathrm{A} 2$.

Finally, we asked the participants after each session and binaural configuration to indicate where and how many sound images they perceived by selecting the images on a drawing of a head. We did not restrict the maximum number of images that the participants could select.

\section{B. Results}

\section{Balanced level}

The results of the adaptive and adjustment procedure of Experiment 1, averaged per participant across four sessions, are shown in Fig. 1. For normal hearing listeners, we expect a balanced level at the comparison ear of $60 \mathrm{~dB}$ A. As can be seen in the figure, the averaged median value across four different binaural configurations of the adjustment procedure was closer to this level compared to that of the adaptive procedure. The median values of for the four test stimuli were as follows: correlated stimuli configuration $(60.5 \mathrm{~dB}$ for the adjustment vs $60.0 \mathrm{~dB}$ for the adaptive procedure), uncorrelated stimuli configuration $(59.6 \mathrm{~dB}$ for the adjustment vs $58.3 \mathrm{~dB}$ for the adaptive procedure), small shift stimuli configuration $(56.3 \mathrm{~dB}$ for the adjustment vs $52.0 \mathrm{~dB}$ for the adaptive procedure), big shift stimuli configuration (57.9 dB for the adjustment vs $56.3 \mathrm{~dB}$ for the adaptive procedure).

Since the results were not normally distributed and had unequal variances, we performed a three-way robust analysis of variance (ANOVA) with 20\% trimmed means (Wilcox, 


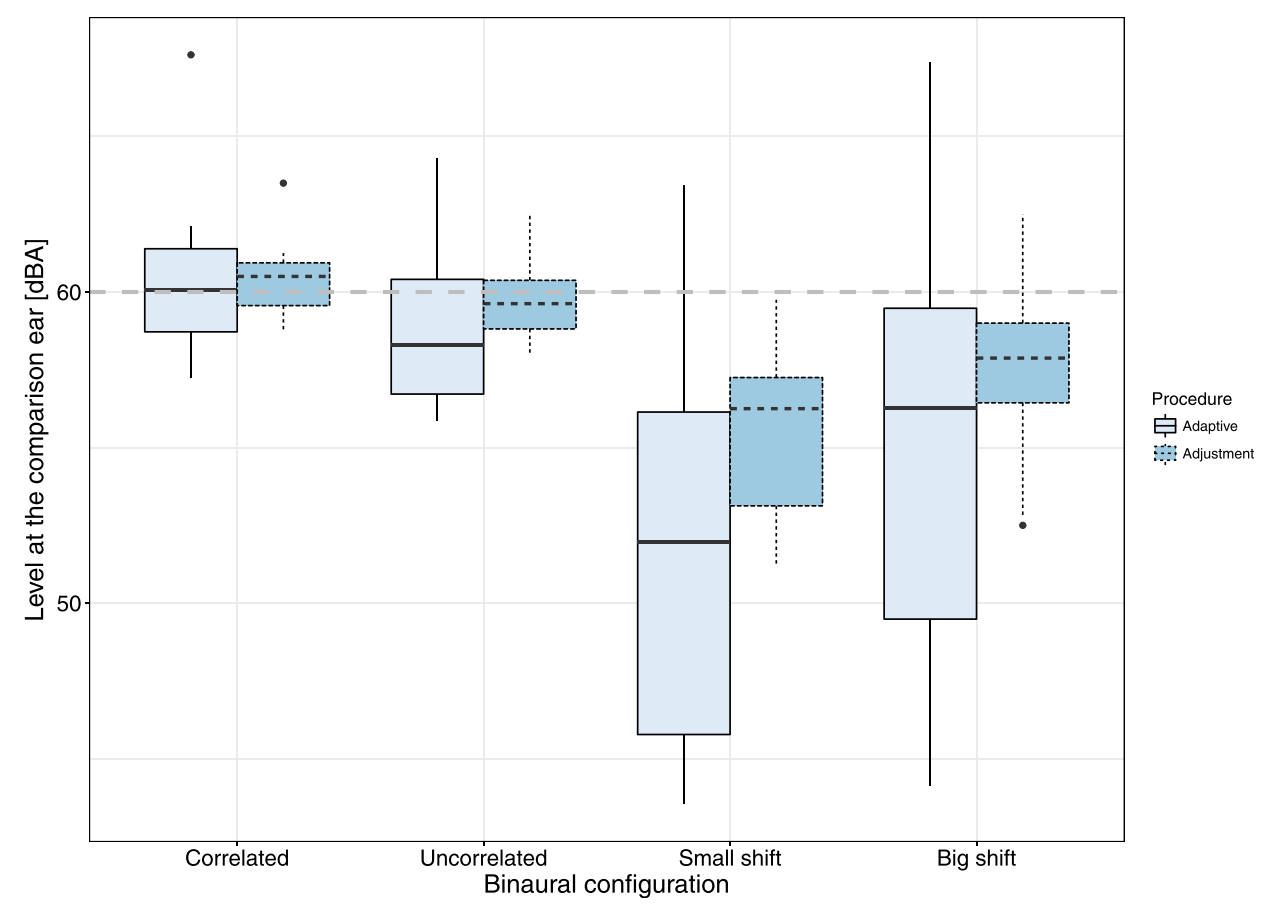

FIG. 1. (Color online) The results of the adaptive and adjustment procedure of Experiment 1, averaged per participant across four sessions. The horizontal dashed line refers to the level at the reference ear.

2012). We used the factors "procedure," "binaural configuration," and "session." We found a significant main effect of binaural configuration $(Q=108.81, p<0.001)$, a significant effect of procedure $(Q=14.6, p=0.001)$, and a significant interaction between procedure and binaural configuration $(Q=11.31, p=0.014)$, which means that with frequency shift the effect of procedure increased. Since the factor session and all interactions with this factor were never significant $(p>0.05)$, for each of the two procedures, the results per participant are averaged across four sessions in Fig. 1.

\section{Variability of balanced levels}

As a metric of reliability, for each participant and each binaural configuration, we calculated the standard deviation of the loudness balanced levels in $\mathrm{dB}$ across the four sessions. The standard deviations in $\mathrm{dB}$ for four different binaural configurations are shown in Fig. 2. As can be seen in the figure, the adjustment procedure had smaller standard deviations than the adaptive procedure.

Wilcoxon signed-rank tests with Holm corrections on standard deviations revealed that for the adaptive procedure, the small shift binaural configuration had significantly larger standard deviations than the correlated [corrected $p=0.018$, $95 \%$ confidence interval $=(-3.4 ; 1.5)]$ and uncorrelated [corrected $p=0.001,95 \%$ confidence interval $=(-3.0$; -1.2)] binaural configurations. Additionally, the tests revealed that for the uncorrelated [corrected $p=0.032,95 \%$ confidence interval $=(-0.5 ; 1.7)]$ and small shift $[$ corrected $p=0.032,95 \%$ confidence interval $=(1.1 ; 2.8)]$ binaural configuration, the adaptive procedure had significantly larger standard deviations than the adjustment procedure.

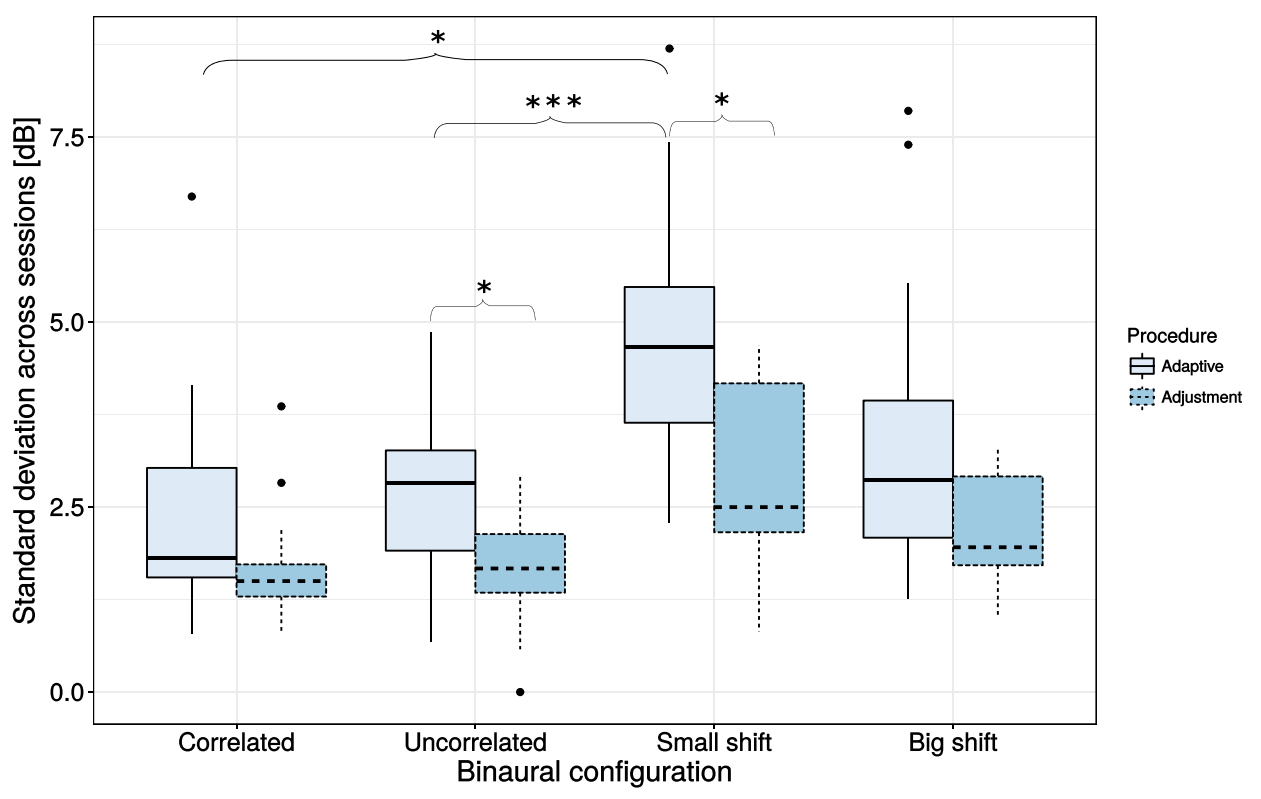

FIG. 2. (Color online) Standard deviation across sessions of the results for each subject for four binaural configurations and procedures. 


\section{Just noticeable differences in ILD}

To investigate possible correlations between the variability of the results and the participants' JNDs, we estimated the JNDs in ILD from the results of our adaptive procedure. To estimate the JND for each participant, we calculated the difference of the averaged levels of the 2-down 1-up and the 1down 2 -up sequence, which are targeting $71 \%$ and $29 \%$ of the psychometric function.

The mean JND for each subject and each binaural configuration is shown in Fig. 3. As seen in Fig. 3, the JNDs are larger when there is a frequency shift between the binaural stimuli, which was also confirmed by the statistics shown below.

We performed a two-way robust ANOVA with 20\% trimmed means with factors "binaural configuration" and "session" and found a significant main effect of binaural configuration $(Q=75.98, p=0.001)$, no significant effect of test session $(Q=5.07, p=0.197)$, and no interaction between these factors $(Q=4.22, p=0.917)$. Since the effect of session was insignificant, the JNDs were averaged over the four test sessions in the Fig. 3.

Furthermore, we performed Wilcoxon signed-rank tests with Holm corrections on mean JNDs per participant across session. We found that the JNDs for the uncorrelated binaural configuration were significantly greater than the JNDs for the correlated binaural configuration [corrected $p=0.021$, $95 \%$ confidence interval $=(-0.45 ;-0.05)]$. Also, the JNDs for the small frequency shift binaural configuration were significantly greater than the JNDs for the uncorrelated binaural configuration [corrected $p=0.003,95 \%$ confidence inter$\mathrm{val}=(-1.23 ;-0.49)]$. In contrast, there was no significant difference between the JNDs for the small and big frequency shift binaural configurations (corrected $p=0.8$ ).

Finally, we found a significant correlation $(r=0.67$, $p=0.036$ ) between the estimated mean binaural JND (Fig. 3 ) and the standard deviation of the adaptive procedure for the binaural correlated stimuli (the left panel of Fig. 2). For the other binaural configurations the correlations were not significant.

\section{Test duration}

The global medians of the duration of the adjustment and the adaptive procedures were 29 and $155 \mathrm{~s}$, respectively. With regard to the test duration of the adjustment procedure, a two-way robust ANOVA with $20 \%$ trimmed means showed a significant main effect of session $(Q=26.62$, $p=0.001$ ), and a significant effect of binaural configuration $(Q=10.04, p=0.027)$. While significantly different, the median time differences between binaural configurations were small, with medians of $28,25.5,33$, and $28 \mathrm{~s}$, for the correlated, uncorrelated, small shift, and big shift configuration, respectively. From session A1 to session B2, the median duration decreased from 37 to $25 \mathrm{~s}$ per run.

For the adaptive procedure, the same test only showed a significant main effect of session $(Q=33.31, p=0.001)$. From session A1 to session B2, the median duration decreased from 163 to $152 \mathrm{~s}$. Therefore, in general the adjustment procedure was about five times faster than the adaptive procedure if we ignore the time for instructions for the two procedures.

\section{Binaural fusion}

In the field, the methods to measure the degree of binaural fusion and to analyse the results are currently still rapidly evolving. We opted for a conservative approach and simply considered the number of reported sound images (Aronoff et al., 2014; Fitzgerald et al., 2015). If the participants selected only one image across all sessions for a particular stimulus, we interpreted that stimulus as clearly fused. For the binaural correlated and uncorrelated stimuli, three and seven out of 14 participants indicated more than one auditory image at least in one session, respectively. In contrast, for

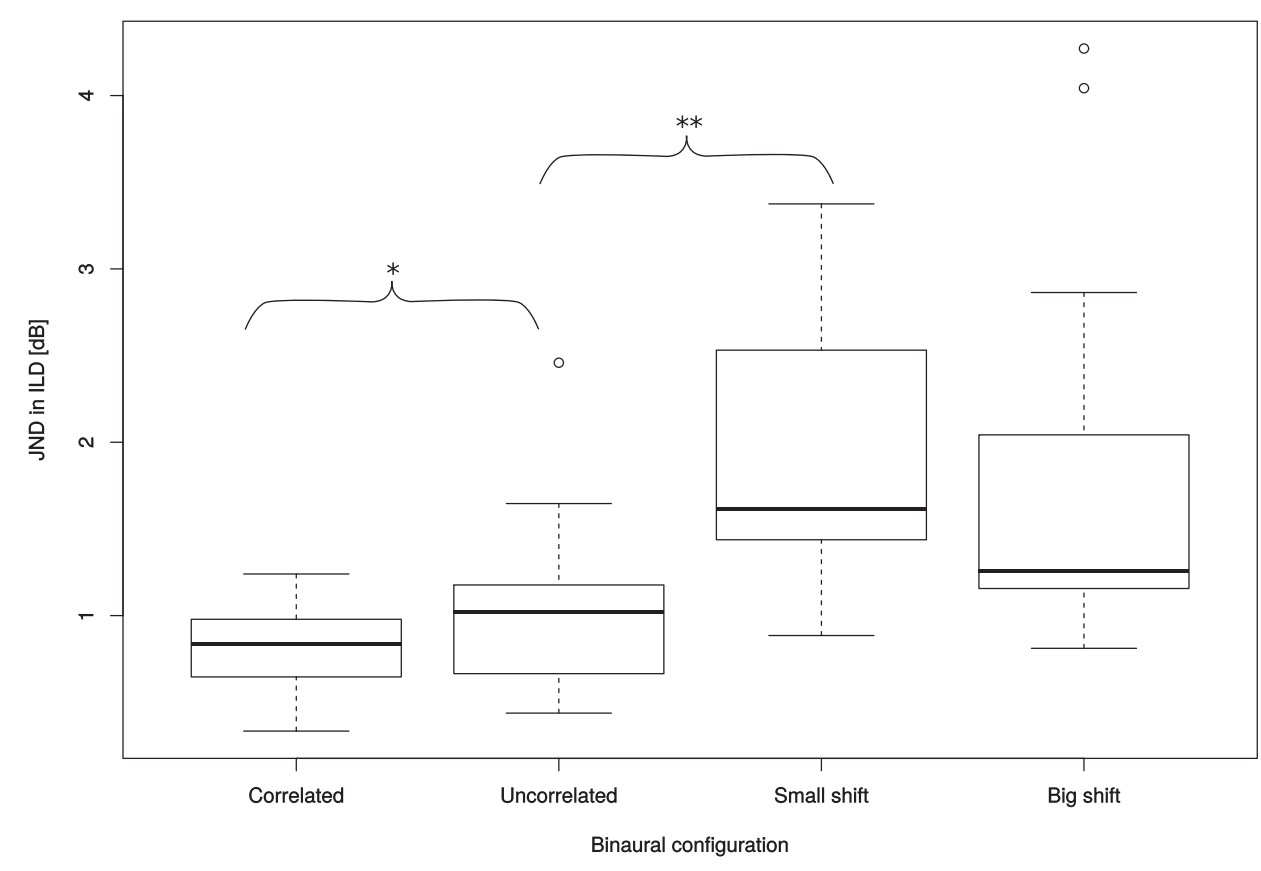

FIG. 3. Just noticeable difference in interaural loudness difference, calculated as the difference between the two sequences of the adaptive loudness balance procedure. 
the small and big frequency shifts, all participants reported more than one auditory image across all sessions.

\section{EXPERIMENT 2}

In Experiment 1, we found better repeatability of the adjustment procedure compared to the adaptive procedure. However, it was unclear whether there was a potential effect of the starting level of the adjustment procedure, which in real bimodal listeners depends on the initial loudness difference between the two devices. Also, further comparison with a larger group of subjects for different reference levels and frequencies was needed in order to generalise the results of Experiment 1.

Therefore, in the second experiment, the two procedures were again compared, using a larger group of subjects. We also investigated the influence of the start level of the adjustment procedure, which was $60 \mathrm{~dB}$ A for both the starting level and the reference level in Experiment 1. Therefore, we investigated the outcome of the adjustment procedure when one of the hearing devices does not deliver a comfortable loudness. The stimuli were different to those used in Experiment 1, and only the correlated configuration was examined. The data were collected as part of a larger study.

\section{A. Methods}

\section{Apparatus}

The apparatus was similar to Experiment 1, but this experiment took place in a soundproof booth and the stimuli were presented binaurally to the participants through Etymotic Research ER-3 A insert ear phones.

\section{Stimuli}

The stimuli were calibrated in dB SPL using a 2 cc Brüel \& Kjær coupler type 4152 and were sinusoidally amplitudemodulated (SAM) pure tones with a modulation frequency of $40 \mathrm{~Hz}$ and a carrier frequency of either 500,1000 , or $2000 \mathrm{~Hz}$. The left, designated as the reference ear, always had a fixed intensity. This intensity was either 50 or $70 \mathrm{~dB}$ SPL for the first group (either 500 or $2000 \mathrm{~Hz}$ carrier) and was always $60 \mathrm{~dB}$ SPL for the second group $(1000 \mathrm{~Hz}$ carrier). All stimuli had a duration of $1 \mathrm{~s}$. For each run the same stimulus (except for the level) was simultaneously presented to the right, designated as the comparison ear, and the reference ear.

\section{Participants}

All 38 participants ( 28 female, 10 male), with an average age of $22 \pm 2$ years, had normal hearing in both ears, as assessed by pure tone audiometry, except one participant who had a threshold of $35 \mathrm{~dB} \mathrm{HL}$ at $500 \mathrm{~Hz}$ in both ears. Two groups were tested. The first group of 19 participants performed the tasks for the 500 and $2000 \mathrm{~Hz}$ stimuli, while the second group of 19 participants performed the tasks for the $1000 \mathrm{~Hz}$ stimuli.

\section{Procedures}

For each stimulus (carrier frequency and reference level), a more extended adjustment procedure was conducted, as well as an adaptive loudness balancing procedure. The two procedures were presented in random order to each participant. Within each procedure, the different stimuli were also presented in random order. We no longer included a training phase, as we did not find any effect of the session in Experiment 1.

During the more extended adjustment procedure, the participants were instructed to find the balanced loudness between the ears. A graphical representation of the procedure is shown in Fig. 4.

A right or left button press ("> $>$ or " $<$ "), which was controlled by the experimenter, resulted in an increase or decrease of $1 \mathrm{~dB}$ in the comparison ear. The participants were instructed to find the balanced loudness by asking the experimenter to push one of the two buttons until they were convinced that the levels at the two ears were balanced. To investigate the influence of the start level of the adjustment procedure from Experiment 1, in this experiment, the start level of the first track was $10 \mathrm{~dB}$ below the fixed intensity of the reference ear. In the beginning, participants always asked the experimenter to increase the level at the comparison ear (i.e., "Up" track as shown in Fig. 4). They were carefully encouraged to explore until they were sure that the sounds were balanced between the two ears. The experimenter enforced at least two reversals around the balanced loudness percept. To focus the participants on the task of finding a centered percept, they were also asked to indicate the location of the stimulus in the head, with the use of a drawing of a circle, that signified a top view of the head.

After finding the balanced loudness, the participants were instructed to indicate the first point of unbalanced loudness, i.e., the JND in ILD, while the experimenter pressed the button in the direction of increasing intensity. While changing the level the in one direction, the participants indicated when the loudness was clearly no longer balanced. The experimenter changed the level of the stimulus in the same direction until the participants indicated on the schematic head that the stimulus was fully lateralized towards one side of the head.

From that stimulus level of the comparison ear (well above the level of the reference ear), the participants were asked to find the balanced loudness level again, as well as the JND of this opposite direction (i.e., "Down" track), while further carefully encouraging the participants to explore until they were sure that the sounds were balanced between the two ears.

The adaptive procedure was similar to the one used in Experiment 1. A 2AFC procedure without feedback was performed with two interleaved independent sequences. The first sequence had a 2-up 1-down decision rule and the second sequence had a 2-down 1-up decision rule, respectively converging to $29 \%$ and $71 \%$ correct. The participant was instructed to indicate which ear was louder, i.e., the left or the right ear. The starting intensity of each sequences was $\pm 10 \mathrm{~dB}$ of the reference intensity. The initial step size was $6 \mathrm{~dB}$. After two and four reversals, it was changed to 3 and $2 \mathrm{~dB}$, respectively. Since we found stable results after 15 trials in Experiment 1, this number of trials was used for each sequence. As in Experiment 1, the average intensity in 


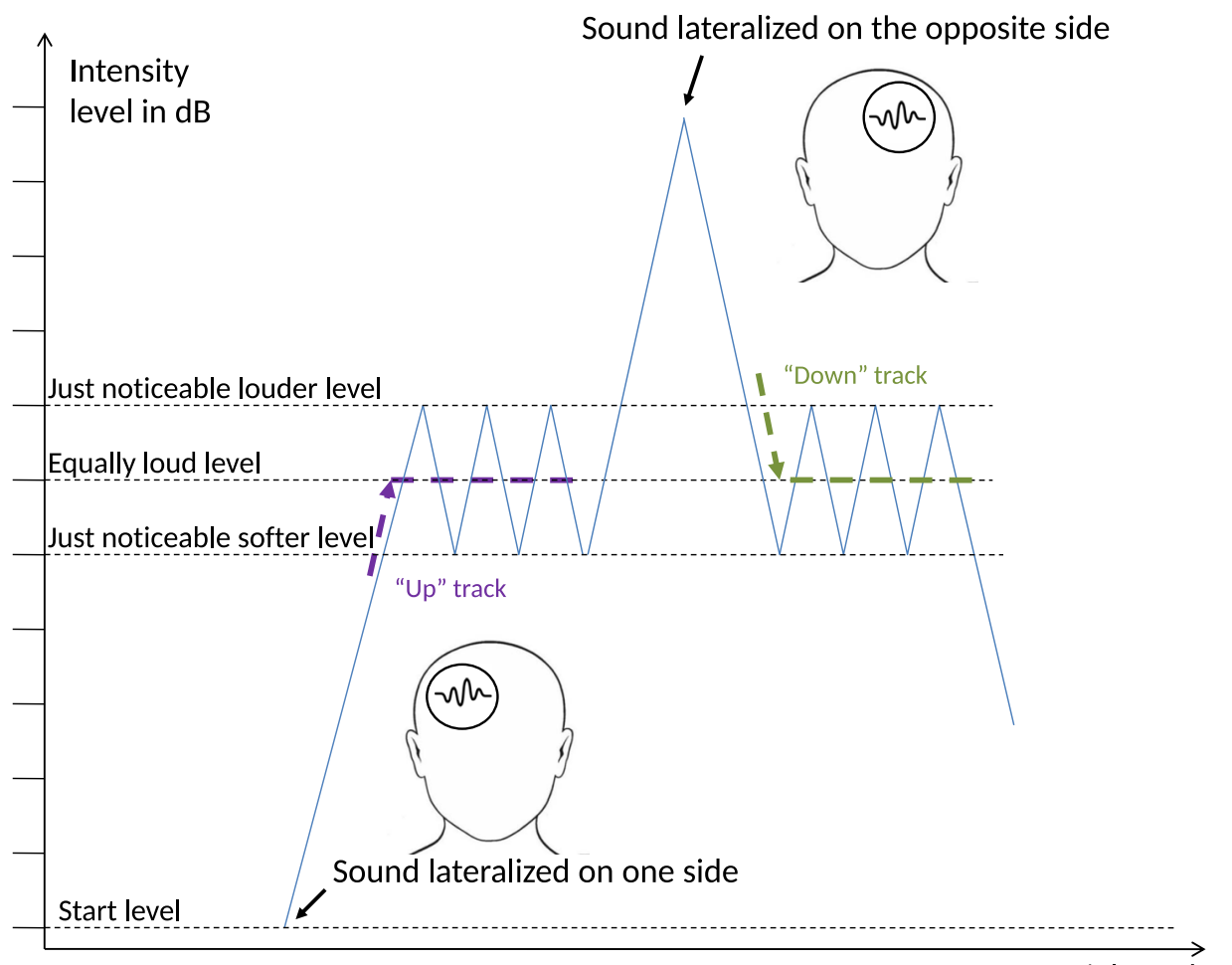

FIG. 4. (Color online) A graphical representation of the extended adjustment procedure.

$\mathrm{dB}$ of the last 12 trials was calculated, and the final balanced intensity was calculated as the average of these values of both sequences.

\section{B. Results}

The results of Experiment 2 are shown in Fig. 5. The levels of the comparison ear corresponding to balanced binaural loudness are shown for different carrier frequencies and levels of the reference ear. As expected, the results obtained from the first, 2-up 1-down staircase differed from the results of the second, 1-up 2-down staircase. Interestingly, a similar trend was observed for the results obtained from the adjustment Up and Down procedure, even though the participants were encouraged to find the equal loudness after each of these parts. In general, the balanced levels found with the two adaptive staircases were similar to the Up and Down part of the adjustment procedure. The final balanced levels of both procedures, i.e., the mean of both staircases of the adaptive procedure and the mean of the Up and Down part of the adjustment procedure, were also very similar. For the adjustment procedure, it seemed that the balanced level was slightly biased towards the side of which the corresponding track began, being either below or above the equal loudness percept.

To test these observations, we first conducted a paired Wilcoxon signed-rank test on the final balanced levels of the adjustment and adaptive procedure and could not find any significant differences between procedures $[V=2638$, $p=0.184$, and a $95 \%$ confidence interval of $(-0.15 ; 0.95)]$. The differences between the first and second staircase of the adaptive procedure, as well as the differences between the Up and Down part of the adjustment procedure, were further explored by conducting paired Wilcoxon signed-rank tests.
As expected, the result of the first staircase of the adaptive procedure was significantly lower than the result of the second staircase $[V=79.5, p<0.001$, with a $95 \%$ confidence interval of $(-2.12 ;-1.58)]$. Interestingly, the result of the Up part of the adjustment procedure was also significantly different from the result of the Down part of the adjustment procedure $[V=432.5, p<0.001$, with a $95 \%$ confidence interval of $(-3.00 ;-2.00)]$.

In summary, it was important to conduct the adjustment procedure twice: once starting from above the balanced level, and once starting from below the expected balanced level in order to prevent a systematic bias towards the starting level. The results of the Up and Down part of the adjustment procedure were similar to the results of the first and second staircase of the adaptive procedure, converging to $29 \%$ and $71 \%$ correct. However, if the adjustment procedure was conducted twice as described above, the final loudness balanced levels of the adjustment procedure were not significantly different from the final loudness balanced levels of the adaptive procedure.

\section{DISCUSSION}

\section{A. Psychophysical procedures}

In both experiments, the adjustment and adaptive procedure yielded similar final loudness balanced levels, and the results remained stable over different test sessions. However, the adjustment procedure was faster and also more reliable given the smaller within subject standard deviations on the balanced levels. The adjustment procedure was slightly biased depending on whether the initial level was above or below the balanced loudness. When the adjustment procedure was conducted twice, starting from opposite perceptual levels, the bias disappeared. 


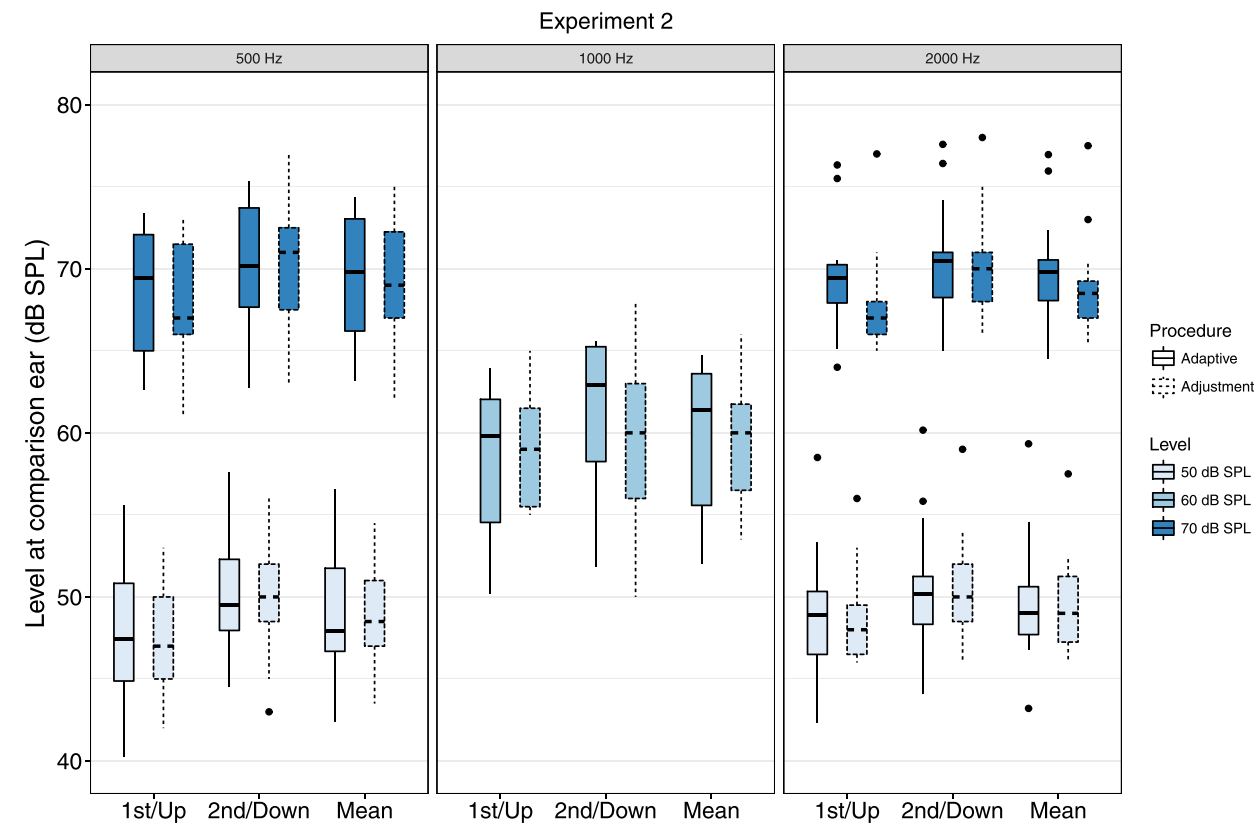

FIG. 5. (Color online) The levels of the comparison ear corresponding to balanced binaural loudness, as found in Experiment 2 for different carrier frequencies and levels of the reference ear. For the adaptive procedure, the results of the first and second staircase, converging to $29 \%$ and $71 \%$ correct, are shown, as well as the mean results of both staircases. For the adjustment procedure, the results of the Up and Down part of the procedure are shown, as well as the mean of both parts of the procedure, corresponding to the final balanced level.

A single run of the (dual-staircase) adaptive procedure took around five times as much time as a run of the adjustment procedure. While the adjustment procedure needs to be conducted twice to avoid bias, and more time is needed for instructing the subject, overall, the adjustment procedure was still faster.

\section{B. Implications for bimodal hearing}

It is known that bimodal users have larger JNDs in level and a larger variation in their JNDs in level than normal hearing participants (Francart et al., 2008a). In order to correctly determine the final step size of the adaptive procedure, it might be necessary to estimate the JND of the participant. However, due to the JND variability, this seems to be difficult for bimodal users. Consequently, the adjustment procedure seems to be better suited.

In Experiment 1, the JNDs increased with increasing frequency mismatch, which corresponds to the results of Francart and Wouters (2007). We also found that the standard deviations of the adaptive procedure were larger when frequency shift was introduced, showing that increased difficulty in ILD perception led to increased variability in the adaptive procedure. Finally, we also found a significant correlation between the JND and the standard deviation of the results of the adaptive procedure, when the two noises were correlated between both ears.

The JNDs in ILD obtained in Experiment 1 corresponded well with the results of Mills (1960), Jesteadt et al. (1977), and Yost and Dye (1988), who found for normalhearing participants JNDs of $0.5-1 \mathrm{~dB}, 0.43-1.57 \mathrm{~dB}$, and $0.7-1.2 \mathrm{~dB}$, respectively, with variations depending on the frequencies and levels.

Introducing a frequency mismatch, which is typical for bimodal listeners (Francart and McDermott, 2013), led to larger deviations from the fixed level of the reference ear and an increase in the variability of the balanced levels for both procedures. However, this variability was smaller for the adjustment procedure.

We found that a frequency mismatch with our stimuli often led to decreased fusion, where we define fusion as whether the subjects perceived one single sound image. Note that in the literature, different types of fusion are defined: formant fusion (Guérit et al., 2014), pitch fusion (Reiss et al., 2016; Tan et al., 2017), percentage of fused sound images (Aronoff et al., 2014; Fitzgerald et al., 2015). One possible reason for the larger variability in balancing result in the small and big shift binaural configurations is the lack of fusion. In bimodal hearing, it could be caused by the frequency mismatches and the different modes of stimulation (acoustical vs electrical). It seems to be difficult to compare two sound images in levels or in positions in the head when the modes of stimulation are different. In previous experiments, subjects also had similar difficulties in pitch matching (Peters et al., 2016) or in vowel integration tasks (Guérit et al., 2014).

\section{CONCLUSIONS}

The adjustment procedure was at least as repeatable as the adaptive procedure. We found bigger JNDs in ILD, when introducing frequency mismatches between ears. Also, in this case, the standard deviation between sessions was larger.

With the adjustment procedure, we found an influence of start level on the result. When the adjustment procedure was repeated from the opposite perceptual side and the result was averaged across the two runs, the bias disappeared.

\section{ACKNOWLEDGMENTS}

M.V.E. was supported by a Ph.D. grant for Strategic Basic Research by the Agency for Innovation by Science and Technology in Flanders (IWT, 131106). D.S. was supported by the Agency for Innovation by Science and Technology in Flanders and Cochlear Ltd (IWT Baekeland, 


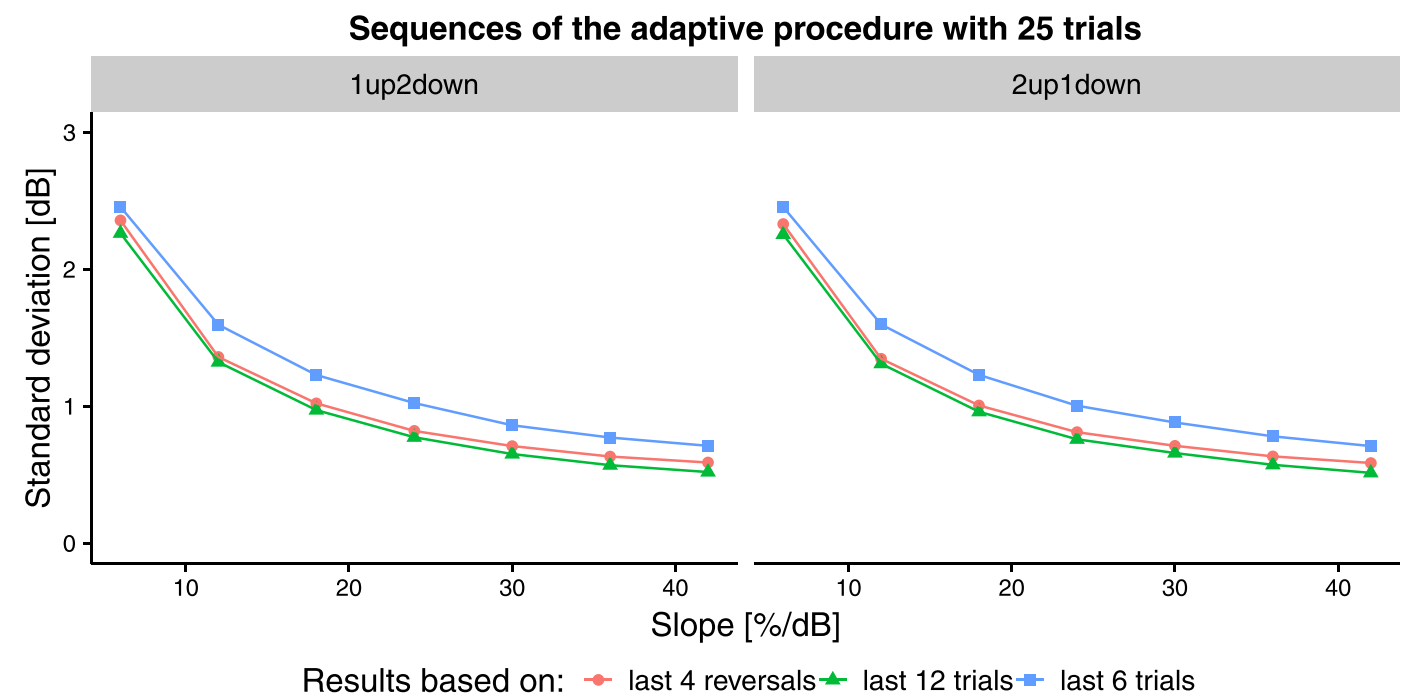

FIG. 6. (Color online) Standard deviation across 10000 Monte Carlo simulations of the sequences of the adaptive procedure as a function of the slope of the psychometric function.

140748). We also thank Dr. V. Best and the two anonymous reviewers for their constructive remarks to improve the manuscript. We are grateful to the participants who voluntarily participated in the experiments, and to I. Bernaerts, E. Meeussen, C. Simons, and J. Vanderydt for their help in collecting some of the data. The authors report no conflict of interest. We thank L. Van Deun for her comments on an earlier version of the manuscript. M.V.E. and D.S. contributed equally to this work and should be considered joint first authors.

\section{APPENDIX: MONTE CARLO SIMULATIONS OF THE EFFECT OF ADAPTIVE PROCEDURE PARAMETERS}

To investigate the difference in outcome between averaging a number of trials or a number of reversals of the sequences of the adaptive procedure, we conducted Monte Carlo simulations. We simulated ideal listeners with different slopes of the psychometric function based on the work of Brand and Kollmeier (2002). We ran the simulation 10000 times for each listener with different slope of the psychometric function. Then, for each sequence, we computed the result as the average of either the last four reversals, or the last 12 trials, or the last six trials. Finally, across all 10000 simulations, we computed the standard deviation of the results as a function of the slope of the psychometric function, as a measure of testretest reliability. The results are shown in Fig. 6. The standard deviation of the simulations is a bit smaller than that of the real experiments, since the simulations do not include lapsing of attention. We concluded that the reliability of the results of the adaptive procedure do not strongly depend on whether we base the result on the last four reversals or the last 12 trials, with a small apparent benefit for the 12 trials.

Some listeners demonstrated JNDs below the step size of the adaptive procedure. While this is not a problem per se, as more than a single trial of the adaptive procedure is used to calculate the final result, it could have slightly affected the precision. We investigated this by conducting Monte Carlo simulations with a step size of $1 \mathrm{~dB}$. Monte Carlo simulations showed that a final step size of $1 \mathrm{~dB}$ would additionally decrease the standard deviation by $0.1 \mathrm{~dB}$, compared to a step size of $2 \mathrm{~dB}$.

Akeroyd, M. (2006). "The psychoacoustics of binaural hearing," Int. J. Audiol. 45, 25-33.

Aronoff, J. M., Shayman, C., Prasad, A., Suneel, D., and Stelmach, J. (2015). "Unilateral spectral and temporal compression reduces binaural fusion for normal hearing listeners with cochlear implant simulations.," Hear. Res. 320, 24-29.

Brand, T., and Kollmeier, B. (2002). "Efficient adaptive procedures for threshold and concurrent slope estimates for psychophysics and speech intelligibility tests," J. Acoust. Soc. Am. 111(6), 2801-2810.

Ching, T., van Wanrooy, E., and Dillon, H. (2007). "Binaural-bimodal fitting or bilateral implantation for managing severe to profound deafness: A review.," Trends Amplif. 11(3), 161-192.

Dorman, M. F., Loiselle, L., Stohl, J., Yost, W. A., Spahr, A., Brown, C., and Cook, S. (2014). "Interaural level differences and sound source localization for bilateral cochlear implant patients.," Ear Hear. 35(6), 633-640.

Fitzgerald, M. B., Kan, A., and Goupell, M. J. (2015). "Bilateral loudness balancing and distorted spatial perception in recipients of bilateral cochlear implants," Ear Hear. 36(5), e225-e236.

Fletcher, H., and Munson, W. (1933). "Loudness, its definition, measurement and calculation," J. Acoust. Soc. Am. 5(2), 82-108.

Francart, T., Brokx, J., and Wouters, J. (2008a). "Sensitivity to interaural level difference and loudness growth with bilateral bimodal stimulation," Audiol. Neurotol. 13(5), 309-319.

Francart, T., and McDermott, H. (2013). "Psychophysics, fitting, and signal processing for combined hearing aid and cochlear implant stimulation," Hear. Res. 34(6), 685-700.

Francart, T., van Wieringen, A., and Wouters, J. (2008b). "APEX 3: A multi-purpose test platform for auditory psychophysical experiments," J. Neurosci. Methods 172(2), 283-293.

Francart, T., and Wouters, J. (2007). "Perception of across-frequency interaural level differences," J. Acoust. Soc. Am. 122(5), 2826-2831.

Guérit, F., Santurette, S., Chalupper, J., and Dau, T. (2014). "Investigating interaural frequency-place mismatches via bimodal vowel integration," Trends Hear. 18, 1-10.

Hoth, S. (2007). "Indication for the need of flexible and frequency specific mapping functions in cochlear implant speech processors," Eur. Arch. Otorhinolaryngol. 264(2), 129-138.

Jansen, S., Luts, H., Dejonckere, P., van Wieringen, A., and Wouters, J. (2013). "Efficient hearing screening in noise-exposed listeners using the digit triplet test," Ear Hear. 34(6), 773-778.

Jesteadt, W. (1980). "An adaptive procedure for subjective judgments," Percept. Psychophys. 28(1), 85-88. 
Jesteadt, W., Wier, C., and Green, D. (1977). "Intensity discrimination as a function of frequency and sensation level," J. Acoust. Soc. Am. 61(1), 169-177.

Lecluyse, W., and Meddis, R. (2009). "A simple single-interval adaptive procedure for estimating thresholds in normal and impaired listeners," J. Acoust. Soc. Am. 126(5), 2570-2579.

Levitt, H. (1971). "Transformed up-down methods in psychoacoustics," J. Acoust. Soc. Am. 49, 467-477.

Mills, A. (1960). "Lateralization of high-frequency tones," J. Acoust. Soc. Am. 32(1), 132-134.

Oetting, D., Brand, T., and Ewert, S. D. (2014). "Optimized loudnessfunction estimation for categorical loudness scaling data," Hear. Res. 316, $16-27$.

Peters, J. P., Bennink, E., Grolman, W., and van Zanten, G. A. (2016). "Electro-acoustic pitch matching experiments in patients with single-sided deafness and a cochlear implant: Is there a need for adjustment of the default frequency allocation tables?," Hear. Res. 342, 124-133.

Reiss, L. A., Eggleston, J. L., Walker, E. P., and Oh, Y. (2016). "Two ears are not always better than one: Mandatory vowel fusion across spectrally mismatched ears in hearing-impaired listeners," J. Assoc. Res. Otolaryngol. 17(4), 341-356.

Salloum, C., Valero, J., Wong, D., Papsin, B., van Hoesel, R., and Gordon, K. (2010). "Lateralization of interimplant timing and level differences in children who use bilateral cochlear implants.," Ear Hear. 31(4), 441-456.
Scollie, S. D., Ching, T. Y., Seewald, R. C., Dillon, H., Britton, L., Steinberg, J., and King, K. (2010). "Children's speech perception and loudness ratings when fitted with hearing aids using the DSL v. 4.1 and the NAL-NL1 prescriptions," Int. J. Audiol. 49, S26-S34.

Stevens, S. S., and Galanter, E. H. (1957). "Ratio scales and category scales for a dozen perceptual continua," J. Exp. Psychol. 54(6), 377-411.

Tan, C.-T., Martin, B., and Svirsky, M. A. (2017). "Pitch matching between electrical stimulation of a cochlear implant and acoustic stimuli presented to a contralateral ear with residual hearing," J. Am. Acad. Audiol. 28(3), 187-199.

Tyler, R., Parkinson, A., Wilson, B., Witt, S., Preece, J., and Noble, W. (2002). "Patients utilizing a hearing aid and a cochlear implant: Speech perception and localization," Ear Hear. 23(2), 98-105.

van Wieringen, A., and Wouters, J. (2001). "Comparison of procedures to determine electrical stimulation thresholds in cochlear implant users," Ear Hear. 22(6), 528-538.

Wier, C. C., Jesteadt, W., and Green, D. M. (1976). "A comparison of method-of-adjustment and forced-choice procedures in frequency discrimination," Percept. Psychophys. 19(1), 75-79.

Wilcox, R. (2012). Introduction to Robust Estimation and Hypothesis Testing, 3rd ed. (Academic Press, New York), p. 608.

Yost, W. A., and Dye, R. H. (1988). "Discrimination of interaural differences of level as a function of frequency," J. Acoust. Soc. Am. 83(5), 1846-1851. 